\title{
Lepidoptera of the Taymyr peninsula, northwestern Siberia
}

\author{
Mikhail V. Kozlov, Jaakko Kullberg \& Vladimir V. Dubatolov
}

Kozlov, M. V., Kullberg, J. \& Dubatolov, V. V. 2006: Lepidoptera of the Taymyr peninsula, northwestern Siberia. - Entomol. Fennica 17: 136-152.

The annotated list of 155 species of Lepidoptera, in addition to five records on unidentified species with known generic affinity, from the Taymyr national district is the first comprehensive account from this area. It is based on the results of the collecting trip of 2002, and on old materials collected by different researchers, and all published records collected by intensive bibliographic search and through consultations with colleagues. Four species erroneously reported from Taymyr are excluded from the list; 65 species are new for this district, including Clepsis mehli that is new to Russia and East Palaearctic, and Argyroploce mengelana reported from Asia for the first time. Records of 32 species are based exclusively on earlier publications; occurrence of other species is confirmed by the investigated material (listed in the paper). We expect that some hundreds of species are still yet to be discovered in Taymyr. The fauna of moths and butterflies of Taymyr is clearly more similar to that of East Palaearctic or Polar Ural than to that of northwestern Europe. In an European perspective, this fauna looks quite exotic and may resemble one that existed in Europe during the ice ages.

M. V. Kozlov, Section of Ecology, University of Turku, FI-20014 Turku, Finland; E-mail:mikoz@utu.fi

J. Kullberg, Finnish Museum of Natural History, Division of Entomology, P. O. Box 17, FI-00014 University of Helsinki, Finland; E-mail:jaakko.kullberg@helsinki.fi

V. V. Dubatolov, Siberian Zoological Museum, Institute of Animal Systematics and Ecology, Frunze street 11, Novosibirsk, 630091 Russia; E-mail: vvdubat @online.nsk.su

Received 27 May 2005, accepted 6 October 2005

\section{Introduction}

Although the first butterflies have been collected in Taymyr (frequently misspelled as "Taimyr") already by A. T. Middendorff, who visited this region during his expedition in 1842-1845 (Ménétriès 1851), the territory of Taymyr peninsula so far remains almost unexplored but faunistically most interesting spot for an entomologist. According to Pagenstecher (1901), only one publication from the $19^{\text {th }}$ century (Trybom 1878) contained data from this arctic region that was thus much less explored than the territories both west (Yamal and Gydan Peninsula) and east of it (Yakutia). This fact is partially explained by the inaccessibility of this area, especially before 1930. For example, the so-called "Arctic collections" of moths and butterflies by N. Y. Kusnezov (some 50 large insect drawers kept in the Zoological Institute of the Russian Academy of Sciences in St. Petersburg) contained only 53 specimens of nine species collected in Taymyr before 1930, 
mainly during the expedition in 1928 led by A. I. Tolmachev.

Entomological results of several expeditions arranged between late 1920s and early 1930s have not been published, and the systematic exploration of the insect fauna of the Taymyr peninsula started in early 1970 s. Several species of butterflies and moths are mentioned in ecologically oriented studies (Chernov 1973, 1978), but more or less comprehensive species lists exist only for butterflies (Korshunov et al. 1982, 1985). Also several species of geometrid moths (Vasilenko 1990, 1995, Viidalepp 2001), tiger moths (Dubatolov \& Zolotarenko 1990, Dubatolov et al. 1997) and noctuids (Zolotarenko 1970, 1990, Klyuchko 1985, 1988, Kononenko 1985, 1997, Lafontaine et al. 1983, 1986, 1987a, 1987b) have been reported from this area, while almost no data were published on other groups.

One of the authors (MVK) visited Noril'sk in July-August of 2002, and in association with environmental assessment of pollution impact on terrestrial ecosystems collected some moths and butterflies. Keeping in mind the scarcity of the faunistic data from the extreme north of Siberia, especially the shortage of information on microlepidopterans, we decided to publish the detailed list of the Lepidoptera so far recorded in Taymyr. One of our goals was to summarize the information published in Russian and make it available to international scientific community.

\section{Study area and collections}

\subsection{Localities}

We restricted our list to an administrative unit called "Taymyrskij (Dolgano-Nenetskij) nazional'nyi okrug" that forms the northernmost part of the Krasnoyarskij kraj (= district) of the Russian Federation. This territory (Fig. 1) is bordered by approx. $80^{\circ} \mathrm{E}$ from the West and $112^{\circ} \mathrm{E}$ from the East, and $67-69^{\circ} \mathrm{N}$ from the South, and includes also Severnaya Zemlya archipelago (up to $81^{\circ} \mathrm{N}$; not shown in Fig. 1).

Although we performed detailed search for locality information also by using historical (preSoviet) maps, some of the records containing incomplete information could not be attributed to reasonably small areas. These records are indicated by the question mark; it is possible that some of the unidentified localities are even outside of the study region. Whenever possible, we accounted for changes in place names and used the most recent versions; however, for some of the old localities we found no correspondence on recent maps, and these localities are marked as "old name". Some toponyms were found twice in Taymyr, which makes the situation even more problematic. In particular, there were two villages called "Zaostrovskaya": one on Yenisey, opposite to the mouth of Malaya Kheta river, and another far North of the Pyasino lake. Keeping in mind the extremely low accessibility of sites that are far from Yenisey, we arbitrarily decided to consider the village on the riverside as the collecting locality by Wuorentaus. We use a new toponym "Eremin's hut" (which cannot be found even on the most recent maps) to designate the collecting locality on Southern shore of Lama lake, $120 \mathrm{~km}$ E of Noril'sk.

Spelling of locality names follows The Times Atlas of the World (Times 2003); if the locality is absent in this atlas, we transliterated the most recent version of the Russian name (Noril'sk 1999) following the rules accepted by The Times.

\subsection{Nomenclature and distribution}

The order of families and nomenclature of most of the groups follow Karsholt \& Razowski (1996). We feel it necessary to comment on the use of subspecific names in our paper, especially for butterflies where a huge amount of subspecific and infraspecific names has been (and is still being) introduced by some researchers. We use the subspecific names only in cases where the populations are disjunct and determinable. However, we include references to subspecific affinities along with the names of persons responsible for this determination.

We use square brackets for species that have been reported from Taymyr, but their occurrence in this region is doubtful. Any species whose description does not contain reference(s) to earlier publications is classified as new for the region.

For the zoogeographical analysis, we classified each species according to their occurrence in 


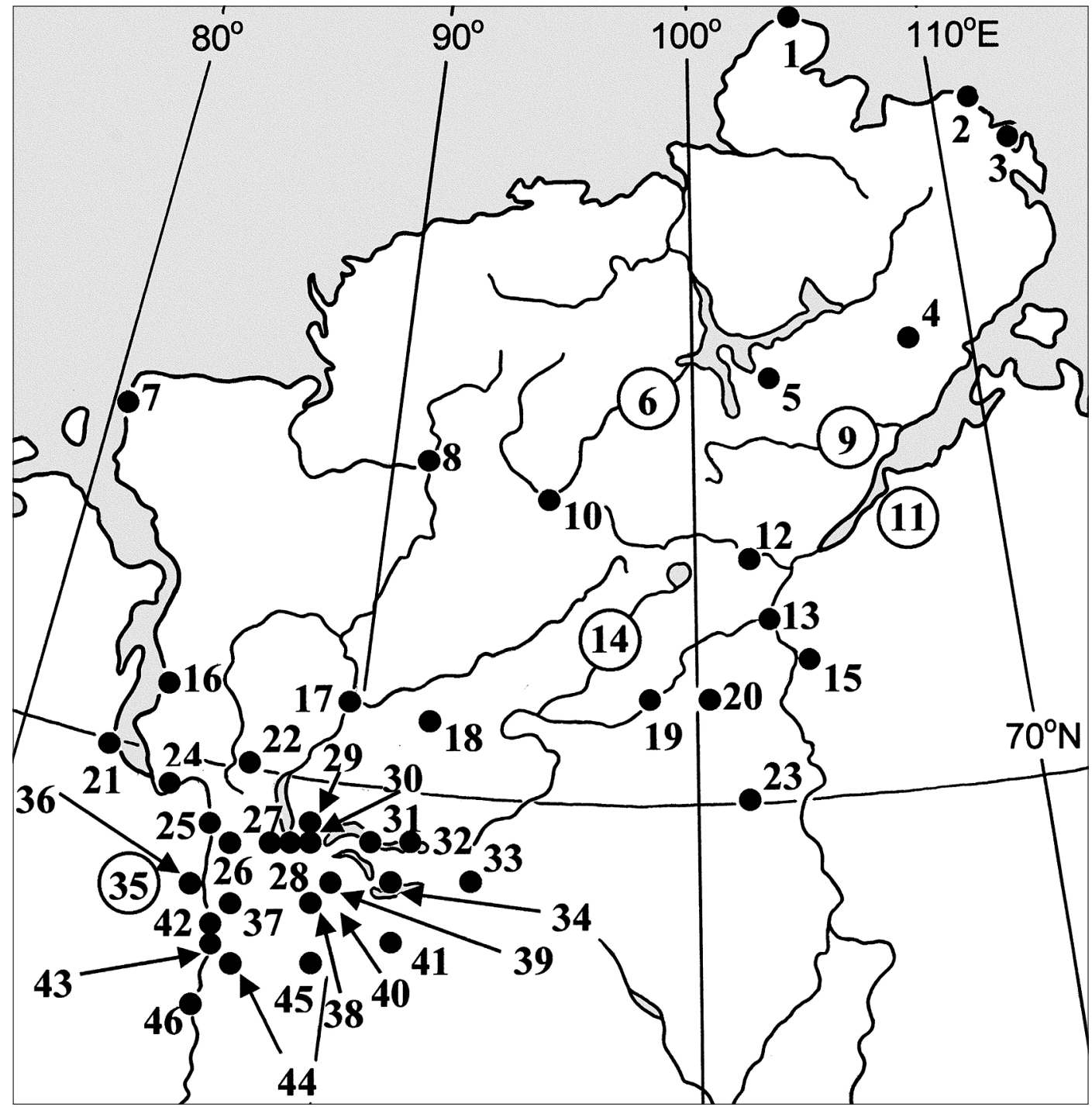

Fig. 1. Collecting localities mentioned in the text. Solid dots = exact locality position; hollow circles = exact position unknown (approximate position provided). Location names (values in parentheses indicate the number of species reported from a given locality): 1 - Chelyuskin cape (1); 2 - Svyatogo Pavla Is. (1); 3 - Dika Bay (1); 4 - Portnyagino lake (2) and Gusikha river (2); 5 - Yamutarida river (1); 6 - Verkhnyaya Taymyra river, low flow (7); 7 - Meduza Bay, 18 km S of Dikson (10); 8 - Tareya (31); 9 - Bol'shaya Balakhnya (1); 10 - Gorbita river mouth (2); 11 - Popigay river (9), Anabarka river (1) and Kunzhalak lake (2); 12 - Ary-Mas, sometimes referred as Novaya river (26); 13 - Khatanga (4); 14 - Boganida river (5); 15 - 25 km S of Kayak (4); 16 - Baikalovsk (1); 17 - Kresty, abandoned village (3); 18 - Chopko river (12); 19 - Bol'shaja Romanikha river (1); 20 - Maymecha river, lower flow (4); 21 - Nosok (9); 22 - Nizhnyaya Agapa river (20); 23 - Maymecha river, upper flow (4); 24 - Zaostrovskaya, abandoned village (4); 25 - Dudinka (25); 26 - Vershininskoe, abandoned village (1); 27 - Kayyerkan (5); 28 - Noril'sk (33); 29 - Talnakh (55); 30 - Valek (2); 31 - Eremin's hut, 120 km E of Noril'sk (22); 32 - Lama tourist centre (27); 33 - Ayan lake, mouth of Ayan river (2); 34 - Sobach'e lake (8); 35 - Pairgakta river (2) and Sokholka lake (1); 36 - Potapovo village (5); 37 - Fokina river, middle flow (5); 38 - Rybnaya river, middle flow (21); 39 - Ketairbe, 20 km from Keta lake (9); 40 - Keta lake (5); 41 - Kutaramakan river, middle flow (7); 42 - Uboinaya river (2); 43 - Khantayka river (17); 44 - Snezhnogorsk (10); 45 - Khantayskoe lake (7); 46 - Igarka (outside Taymyr peninsula). 
three large regions: West and East Palaearctic (separated by the Ural mountains; abbreviated as WP and EP, respectively) and Nearctic. Species recorded in all three regions are classified as holarctic (HA); recorded in both West and East Palaearctic as Palaearctic (PA); recorded in East Palaearctic and Nearctic as Beringian (BE).

\subsection{Material}

Since the data form Taymyr are scarce, we provide the (abbreviated) label information for every investigated specimen, sometimes in a modified form to make the text uniform and easily understandable. In particular, we pooled records from three localities referred by Zolotarenko (1970, 1990) as Talnakh, Listvyanka river and Kharaerlakh river, because the mentioned rivers are within $10 \mathrm{~km}$ from Talnakh and the precise location of the collecting sites cannot be recovered. Similarly, records from Oganer (an eastern suburb of Noril'sk, ca. $8-10 \mathrm{~km}$ from the city center) were attributed to Noril'sk. Collecting dates are sometimes pooled in such a way that only the first and the last collecting day are mentioned. Order of localities within each species follows the order given in Fig. 1, e.g. from North to South, and within approximately the same latitude - from West to East.

Collectors are abbreviated as follows: C-Y.I. Chernov; G-G. A. Grigorjev; K-M. V. Kozlov and V. E. Zverev; Kh-O. A. Khruleva; M - V. V. Mikhailov; M\&B - O. L. Makarova and A. B. Babenko; P-Polovinkina; S - J. Sahlberg; W-Y. Wuorentaus; and Z - G. S. Zolotarenko.

\subsection{Depositories}

Material collected by M. Kozlov is deposited in the Natural History Museum in London. We included data from specimens deposited in Zoological Institute in St. Petersburg, Siberian Zoological Museum in Novosibirsk, the Museum of Natural History of the University of Helsinki and in private collections of G. A. Grigorjev and B. A. Khramov (St. Petersburg). We also investigated samples collected in Taymyr by researchers of A. N. Severtsov Institute of Ecology and Evolution,
Moscow. Although we are aware on materials deposited in the Zoological Museum of the Moscow State University, we were unable either to get access to these collections or agree with the Moscow scientists about the joint publication of the data.

\section{List of species}

Eriocraniidae

Eriocrania sangii (Wood) - Dudinka: 20 4.VII.1980 (Savenkov). PA.

Eriocrania sp. - Noril'sk; Lama: mines on Betula spp., infrequent (K).

Hepialidae

Pharmacis fuscoargenteus (Bang-Haas) Talnakh: $1 \circlearrowleft$ 2.VIII.1964 (Z). HA.

Incurvariidae

Incurvaria circulella (Zett.) - Nizhnyaya Agapa r.: $1 \jmath^{\Im}$ 7.VII.2001 (Kh). The northernmost record; from Eastern Palaearctic so far was known from Khamar-Daban Mts. in Siberia (Kozlov 1996). PA.

Prodoxidae

Greya variabilis Davis \& Pellmyr - Meduza Bay: $1{ }^{1} 1$ 1 15. VII.1998 (Kh); Nizhnyaya Agapa r., $40 \mathrm{~km}$ from the headwater: $1 \lesssim 23$.VII.1973 (Zherikhin). The westernmost records of the species described from North America in 1992 and later on reported from the Chukchi Peninsula (Kozlov 1996). BE.

Tineidae

Monopis weaverella (Scott) - Lama: 1 + 26.28.VII.2002 (K). Cosmopolitan.

Gracillariidae

Phyllonorycter sp. - Lama: mines on the underside of Alnus fruticosa leaves; rare (K).

Plutellidae

Plutella xylostella (L.) - Meduza Bay: 4ð 2 ㅇ 20.VI.-15.VII.2000 (Kh); Noril'sk: 14ð 1 우 21.-29.VII.2002 (K); Talnakh: 1ठ઼ 29.VII. 2002 (K); Eremin's hut: 3 ㄱ 7우 28.VII.2002 (K); Lama: 7ð 26.-28.VII.2002 (K); So- 
bach'e lake: 1 \% 27.VII.-13.VIII.1996 (M\&B). Cosmopolitan.

Plutella hyperboreella Strand - Nizhnyaya Agapa r.: $1 \hat{\jmath}$ 14.VII.2001 (Kh). HA.

Oecophoridae

Schiffermuelleria stipella (L.) - Lama: $3 ð$ 26.28.VII.2002 (K). PA.

Coleophoridae

Coleophora murinella Tengstr. - Lama: $3 \widehat{o}$ 26.28.VII.2002 (K). PA.

Coleophora virgaureae Stt. - Talnakh: 19 29.VII.2002 (K). PA.

Momphidae

Mompha polaris Sinev - Noril'sk: 20ิ 21.25.VII.2002 (K); Ayan: $3{ }^{\lambda}$ 20.VII.1983 (Eskov). Although S. Sinev now considers this species as a synonym of M. idaei (Z.), one of us (JK) believes that at least in Europe these two taxa are clearly separable, especially by external characters. Furthermore, larvae of $M$. polaris fed on Epilobium latifolium, while larvae of M. idaei fed on E. angustifolium. M. polaris was found in Polar Ural in 1994 (J. Kullberg, pers. obs.). BE.

Mompha conturbatella (Hb.) - Noril'sk: $13 \bigcirc 1$ 个 21.-25.VII.2002 (K), by sweep-netting from Epilobium sp. on wastelands near the city. HA.

Scythrididae

Scythris cf. obscurella (Scop.) - Noril'sk: 1ठ 21.-25.VII.2002 (K). The species, although closely related to $S$. obscurella, clearly differs from it in the male genitalia structure. The same taxon was found in Polar Ural ( $\mathrm{J}$. Kullberg, pers. obs.); the shortage of the available material does not allow determination or description of this species. EP.

Gelechiidae

Bryotropha galbanella (Z.) - Nizhnyaya Agapa

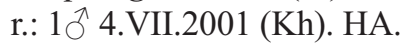

Chionodes lugubrellus (F.) - Lama: $1 \lesssim 1 q$ 26.28.VII.2002 (K). HA.

Chionodes viduellus (F.) - Zaostrovskaya: $1 ð$ 1915 (W). HA.

Acompsia subpunctella Svenss. - Lama: 1ð̋ 26.28.VII.2002 (K). PA.
Tortricidae

Aethes deutschiana (Zett.) - Nizhnyaya Agapa r.: $1 \delta$ 5.VII.2001 (Kh); Khantayka: $1 \delta$ 18.VII. 1876 (S). HA.

Eana osseana (Scop.) - Noril'sk: $2 ð$ 21.-25.VII. 2002 (K); Nizhnyaya Agapa r.: 19 7.VII. 2001 (Kh). HA.

Aphelia septentrionalis Obraztsov - Talnakh: $1 \hat{\sigma}$ 3.VIII.1964 (Z); Noril'sk: 5 ภ 2 + 21.-25.VII. $2002(\mathrm{~K})$. BE.

Clepsis moeschleriana (Wck.) - Talnakh: $1 \hat{\sigma}$ 3.VIII.1964 (Z); Nizhnyaya Agapa r.: $2 ð 2$ 우 13.-16.VII.2001 (Kh). BE.

Eulia ministrana (L.) - Lama: $1 \circlearrowleft 1$ 1 1 26.-28.VII. 2002 (K). HA.

Sparganothis rubicundana (Den. et Schiff.) Dudinka: $1 \widehat{\jmath}$ 26.VII.1876 (S); Noril'sk: $2 \hat{\jmath}$ 1 21.-25.VII.2002 (K); Talnakh: $2 \hat{\jmath}$ 29.VII.2002 (K); Eremin's hut: $1 \jmath^{\lambda} 28$. VII. $2002(\mathrm{~K})$; Lama: $3 \hat{\jmath} 1$ ㅇ 26.-28.VII.2002 (K). HA.

Tia enervana (Erschoff) - Lama: 39 26.-28.VII. 2002 (K). BE.

Argyroploce mengelana (Fernald) - Meduza

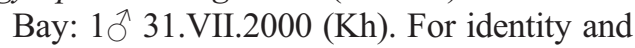
distribution in Nearctic consult Miller \& Jalava (2000). Recently reported from Novaya Zemlya (Aarvik 2004). New to East Palearctic. HA.

Argyroploce lediana (L.) - Nizhnyaya Agapa r.: $1{ }^{\Uparrow}$ 3.VII.2001 (Kh); Lama: $1 \overbrace{}^{\Uparrow}$ 26.-28.VII. 2002 (K). PA.

Phiaris obsoletana (Zett.) - Dudinka: 1ठ 1.12.VII.1915 (W); Zaostrovskaya: $1 \overbrace{}^{\lambda} 1915$ (W); Khantayka: $1 \widehat{\jmath}$ 20.VII.1876 (S); Noril'sk: 1 + 21.-25.VII.2002 (K); Eremin's

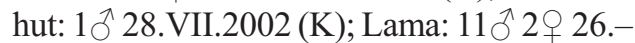
28.VII.2002 (K); Sobach'e lake: $1 ð$ 27.VII.13.VIII.1996 (M\&B). PA.

Phiaris turfosana (Zett.) - Khantayka: $2 \hat{\sigma}$ 16.VII.1876 (S). HA.

Phiaris dissolutana (Stange) - Noril'sk: $1921 .-$ 25.VII.2002 (K); Eremin's hut: 19 28.VII. 2002 (K); Lama: 4ð 26.-28.VII.2002 (K). PA.

Phiaris heinrichana (McDonnough) - Eremin's hut: $2 \widehat{\jmath}$ 28.VII.2002 (K); Lama: 1 q 26.28.VII.2002 (K). HA.

Phiaris inquietana (Walk.) - Ary-Mas: $2 \AA$ 20.28.VII.1983 (P); Tareya (Chernov 1973, 
1978); Nizhnyaya Agapa r.: 2 + 12.14.VII.2001 (Kh). BE.

Selenodes sp. - Meduza Bay: $50 \hat{~} 39$ 8.15.VII.1998 (Kh.).

Zeiraphera diniana (Gn.) - Tareya: migrants from taiga zone (Chernov 1978); Noril'sk: $2{ }^{\top}$ 1 ㅇ 21.-25.VII.2002 (K); Talnakh: $1{ }^{\Uparrow} 1$ 우 29.VII.2002 (K); Eremin's hut: $2 \widehat{\bigcirc} 1$ 우 28.VII.2002 (K); Lama: $2 \hat{\bigcirc} 2$ 2 26.28.VII.2002 (K). PA.

Eucosma guentheri (Tengstr.) - Tareya (Chernov 1973, 1978). PA.

Eucosma tetraplana Möschler - Meduza Bay: 1 + 8.VII.1998 (Kh). EP.

Eucosma ommatoptera Falk. - Meduza Bay: 1 q 8.VII.1998 (Kh). EP.

Dichrorampha montanana Dup. - Talnakh: $5{ }^{\lambda}$ 1 ㅇ 29.VII.2002 (K). PA.

Dichrorampha flavidorsana Knaggs. - Noril'sk: 1ㅇ 21.-25.VII.2002 (K). PA.

Gypsonoma parryana Curt. - Nizhnyaya Agapa r.: 1ठ 4.VII.2001 (Kh). BE.

Clepsis mehli (Opheim) - Nizhnyaya Agapa r.: 2仓 8.-16.VII.2001 (Kh). The species was so far reported from northern Norway only; new to Russia and East Palearctic. PA.

Pterophoridae

Platyptilia calodactyla (Den. et Schiff.) Noril'sk: 1 9 21.-25.VII.2002 (K); Talnakh: $1 q$ 29.VII.2002 (K); Nizhnyaya Agapa r.: $1{ }^{\Uparrow}$ 1 7.-16.VII.2001 (Kh). PA.

Paraplatyptilia sibirica (Zag.) - Tareya: $5 \lesssim 1$ 20.VII.1969 [paratypes: Zagulajev (1983)]; Nizhnyaya Agapa r.: 2今 4.-16.VII.2001 (Kh). EP.

Paraplatyptilia sahlbergi (Poppius) - Dudinka: 1 1 16.VII.1876 (S) (paralectotype); Nizhnyaya Agapa r.: 1 ㅇ 20.VII.1999 (M\&B), $2 \lesssim 3$ 우 4.-16.VII.2001 (Kh). EP, in the west to the Kanin peninsula.

Paraplatyptilia terminalis (Erschoff) Khantayka: $1 \delta^{\lambda} 1876$ (S). EP.

Stenoptilia veronicae Karv. - Noril'sk: 1 + 21.25.VII.2002 (K). PA.

Hellinsia tephradactyla (Hb.) - Noril'sk: 1 + 21.25.VII.2002 (K). PA.

Hellinsia mongolica Zag. \& Pen. - Nizhnyaya Agapa r.: $2 \hat{\jmath} 1$ ㅇ 5.-15.VII.2001 (Kh). EP.
Pyralidae

Catoptria maculalis (Zett.) - Zaostrovskaya: $1{ }^{\lambda}$ 1915 (W); Lama: 1ð 26.-28.VII.2002 (K). HA.

Catoptria trichostoma (Chr.) - Dudinka: $3 \overbrace{}^{\widehat{2}} 2$ 우 1.-12.VII.1915 (W); Nizhnyaya Agapa r.: $1{ }^{\lambda}$ 3 9 5.-16.VII.2001 (Kh). BE.

Pediasia zellerella (Stgr.) - Khantayka: 2 exx. 18.VII.1876 (S); Dudinka: 1 ex. 1.-12.VII. 1915 (W); Lama: 1 26.-28.VII.2002 (K). BE.

Crambus hamellus (Thnbg.) - Sobach'e lake: 1 ㅇ 3.VIII.1996 (M\&B). HA.

Gesneria centuriella (Den. et Schiff.) - Talnakh: 1 `े 29.VII.2002 (K). HA.

Eudonia alpina (Curt.) - Eremin's hut: 2 ㅇ 28.VII.2002 (K); Lama: 1ð 26.-28.VII.2002 (K); Nizhnyaya Agapa r.: 2ð 5.-7.VII.2001 (Kh). The record of Scoparia sp. from Tareya (Chernov 1973) most likely concerns this species. PA.

Udea inquinatalis (Lienig et Z.) - Noril'sk: 10 21.-25.VII.2002 (K); Eremin's hut: 2 ㅇ 28.VII.2002 (K). HA.

Udea torvalis Möschler - Meduza Bay: $6 \AA 7$ ㅇ 7.-17.VII.1998 (Kh). BE.

Udea cretacea Fil. - Talnakh: $2 \hat{\circ} 3$ ㅇ 29.VII.2002 (K). EP.

Hesperiidae

Pyrgus centaureae (Rbr.) - Maymecha r., lower flow: 1 đิ 1982 (Zakharzhevskii); Maymecha r., upper flow: 1ठ 9.VIII.1982 (Zakharzhevskii); Potapovo: 25.VII.1876 (Trybom 1878); Rybnaya r.: $1{ }^{\Uparrow} 12$.VII.1976 (Korshunov et al. 1982); Khantayka: 15.VII.1876 (Trybom 1878). HA.

Carterocephalus palaemon (Pall.) - Chopko r.: 1ð 15.VII.1976 (Chernenko); Rybnaya r.: 1 ठ 18.VII.1976 (Pupavkin); Snezhnogorsk: $1 \precsim$ 22.VII.1980 (Korshunov et al. 1982). HA.

Carterocephalus silvicola (Meig.) - Valek: 1ठ 6.VII.1977 (Korshunov et al. 1982); Eremin's hut: 1 ^ 28.VII.2002 (K); Rybnaya r.: $1 \lesssim$ 18.VII.1976, 1ð2ㅇ 19.-22.VII.1978 (Korshunov et al. 1982); Snezhnogorsk: 1 + 25.VII.1980 (Korshunov et al. 1982). PA.

Papilionidae

Parnassius phoebus (F.) - Talnakh: $2 \widehat{\jmath} 1$ 우 
30.VII.1985 (M), 7ðึ 2 ㅇ 13.-20.VII.2001 (G); Fokina r.: 1 ? early VII.1978 (Godovikova) (Korshunov et al. 1982). HA. Papilio machaon L. - Ary-Mas (Murzin 1982a); Dudinka: 26.VII.1876 (Trybom 1878); Talnakh: several exx. observed on 13.20.VII.2001 (G), 1ठ 23.VII. 1964 (Korshunov et al. 1985); Keta lake: $3 ð 1$ 우 22.VII. 1982 (Korshunov et al. 1985); Kutaramakan r.: $1{ }^{\Uparrow} 1$ 1 12.VIII.1980 (Korshunov et al. 1982). PA.

\section{Pieridae}

Aporia crataegi (L.) - 'Taymyr' (Antonova et al. 1976); Ary-Mas (Lvovsky 1984); 25 km S of Kayak (Lvovsky 1984). PA.

Euchloe creusa kurentzovi Beljaev - Keta lake: $1{ }^{\Uparrow} 22$.VII.1982 [Korshunov et al. (1985), as ssp. orientalis Brem.]; Khantayskoe lake: $1{ }^{\top}$ 15.VII.1974 (M). BE.

Euchloe ochracea Trybom - Ary-Mas: 10 VI.1983 [Korshunov et al. (1985), as E. ausonia]; Kayyerkan: 1ठ 26.VI.1970 [Korshunov et al. (1982), as E. creusa orientalis]; Talnakh: $5 \overbrace{}^{\lambda}$ 4ㅇ 13.-18.VII.2001 (G); Rybnaya r.: $6 \jmath^{\Uparrow} 3$ 12.-22.VII.1978 [Korshunov et al. (1982), as E. creusa orientalis]; Ketairbe r.: 17ð 12 ㅇ 18.-22.VII.1982 [Korshunov et al. (1985), as E. ausonia]; Kutaramakan r.: 19 12.VIII.1980 [Korshunov et al. (1982), as E. creusa orientalis]; Snezhnogorsk: 1 ㅇ 12.VII.1989 [Korshunov et al. 1982, as E. creusa orientalis]; Khantayskoe lake: 1ð 15.VII.1974 (M). EP.

Pieris napi (L.) - Tareya: $2 \hat{\jmath} 3 q$ 17.-21.VII. 1975 (Korshunov et al. 1985); Baikalovsk: 13.-18.IX.1876 [Trybom (1878), as P. napi bryoniae]; Nosok: $2 \hat{\jmath} 1 \uparrow 22 . \&$ 28.VII. \& 7.VIII.1977 (Korshunov et al. 1982); Dudinka: 30.VII.-4.VIII.1876 [Trybom (1878), as P. napi bryoniae], $10 \AA$ 23.VII. 1955, 2ð 11.VII.1977 (Korshunov et al.

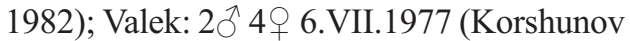
et al. 1982); Talnakh: $2 \hat{\jmath} 1 q$ 29.VII.2002 (K), 4ð 4우 20.-23.VII.1964, $1{ }^{\lambda} 1$ 우 21.VII.1964, 2 1 ㅇ 4.-5.VIII.1964 (Korshunov et al. 1982); Eremin's hut: $2 \hat{\jmath} 3$ ㅇ 28.VII.2002 (K); Lama: $4 \bigcirc^{\lambda} 1$ + 26.28.VII.2002 (K); Potapovo: 25.VII.1876 [Trybom (1878), as P. napi bryoniae];
Pairgakta r.: 1 ㅇ 1.VIII.1966 (Korshunov et al. 1982); Sokholka lake: 3̧े 14.-

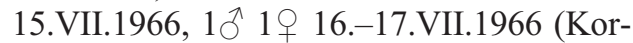
shunov et al. 1982); Fokina r.: 2 early VII.1978 (Korshunov et al. 1982); Rybnaya r.: 7 5 \% 13.-21.VII.1978 (Korshunov et al. 1982); Ketairbe r.: 1ठึ 27.VII.1982 [Korshunov et al. (1985), as ssp. intermedia Krul.]; Snezhnogorsk: 2犬̂ 10.VIII.1965, $10 \hat{\overbrace{}} 6$ 우 22.-25.VII.1980 (Korshunov et al. 1982); Khantayskoe lake: $4{ }^{\Uparrow}$ VIII.1980 (Korshunov et al. 1982). HA.

Pontia callidice nelsoni Edwards - Talnakh: 1 ㅇ 13.VII.2001 (G), 19 5.VIII.1964 (Korshunov et al. 1982); Potapovo: 1 25. VII.1876 (Trybom 1878); Kutaramakan r.: 1ठ 12.VIII. 1980 (Korshunov et al. 1982); Ketairbe r.: 4 ${ }^{\wedge}$ 1 ㅇ 18.-22.VII.1982 (Korshunov et al. 1985); Keta lake: $8 \hat{\jmath} 5$ \% 28.VII.1980 (Korshunov et al. 1982); Khantayskoe lake: 1 đิ VIII.1980 (Korshunov et al. 1982). HA.

Colias palaeno orientalis Stgr. - Verkhnyaya Taymyra r.: 10 17.VIII.1983 (Korshunov et al. 1985); Tareya: 49 23.VII.1975 (Korshunov et al. 1985); Anabarka r.: 1 đ̊ 8.VIII.1967 (Korshunov et al. 1982); Popigay r., low flow: 1ð 18.VII.1967, 1 ð 1ㅇ 27.VII.-1.VIII.1968 (Korshunov et al. 1982); Ary-Mas: 203 đ VII.1983 (Korshunov et al. 1985); Boganida r. (Ménétriès 1851); Nosok: $6 \hat{\jmath} 3$ 3 22.28.VII.1977, 2 ^ 16.-20.VIII. 1977 (Korshunov et al. 1982); Zaostrovskaya: $1 \hat{\jmath} 1915$ (W); Noril'sk: 2ð1q 21.-25.VII.2002 (K); Talnakh: $1{ }^{\lambda}$ 4.VIII.1964 (Korshunov et al. 1982), 6수 7ㅇ 13.-20.VII.2001 (G), 1 ㅇ 29.VII.2002 (K); Eremin's hut: $3 \hat{\jmath} 1$ ㅇ 28.VII.2002 (K); Lama: $2 \hat{\bigcirc}$ 26.VII.3.VIII.2001 (G), $2{ }^{\Uparrow} 2$ ㅇ 26.-28.VII.2002 (K); Pairgakta r., headwater: 1 + 4.VIII.1966 (Korshunov et al. 1982); Rybnaya r.: $2{ }^{\widehat{O}} 20$ 4.-18.VI.1976, $10 \overbrace{}^{\lambda} 1$ ㅇ 15.-23.VII.1978 (Korshunov et al. 1982); Ketairbe r.: $2 \hat{\jmath}$ 27.VII.1982 (Korshunov et al. 1985); Kutaramakan r.: $2{ }^{\Uparrow} 19$ 10.VIII.1980 (Korshunov et al. 1982); Khantayka: $1 \sigma^{\lambda}$ 18.VII.1901 (S); Khantayskoe lake: $3 \hat{\jmath} 1$ ㅇ VIII.1980 (Korshunov et al. 1982). Exact positions of four localities given by Murzin (1982b) cannot be recovered. HA.

Colias tyche herzi Stgr. - 'In tundras of Taymyr': 
20 3 34 ㅇ VII.-VIII.1974/1975 [Korshunov et al. (1985), as C. nastes werdandi Zett.]; Pyasina r. (?): Korshunov et al. (1985), as $C$. nastes werdandi Zett.; Verkhnyaya Taymyra r.: 1 ㅇ 24.VII.1983, $1 ð$ 6.VIII.1983 [Korshunov et al. (1985), as C. nastes werdandi Zett.]; Chopko r.: $1 ð$ 12.VII.1976 [Korshunov et al. (1982), as C. nastes werdandi Zett.]; Talnakh: Chernov (1978), as C. nastes; $1 \delta 3$ ㅇ 13.18.VII.2001 (G). PA.

Colias hyperborea Gr.-Grsh. - Verkhnyaya Taymyra r.: 1 ㅇ 24.VII. 1983, 10 6 .VIII.1983 (Korshunov et al. 1985); Talnakh: 1ठ1ㅇ 9.26.VII.1964 (Korshunov et al. 1982), 90 14 13.-20.VII.2001 (G); Rybnaya r.: 2今 22.-27.VII.1978 (Korshunov et al. 1982). EP.

Colias hecla Lef. - Tareya: $1 \jmath^{\lambda} 3 \uparrow$ 18.24.VII.1969 [Korshunov et al. (1982), as ssp. sulitelma Auriv.; record mentioned by Chernov (1973, 1978)]; Kunzhalak lake: 2 우 22.VII.1967 [Korshunov et al. (1982), as ssp. sulitelma Auriv.]; Popigay r., low flow: $2 \hat{\jmath}$ $3 \bigcirc$ 19.VII.1967, 2ð 1.-9.VIII.1968 [Korshunov et al. (1982), as ssp. sulitelma Auriv.]; Ary-Mas: $3 ð 4$ ㅇ VII.1983 [Korshunov et al. (1985), as ssp. sulitelma Auriv.); Chopko r.: 1ठ 14.VII.1976 [Korshunov et al. (1982), as ssp. sulitelma Auriv.]; Zaostrovskaya: 503 우 1915 (W); Dudinka: 101901 (S); Talnakh: 11 đิ 2 ㅇ 13.-17.VII.2001 (G) (determined as ssp. orientis Wnk. by B. Khramov). The taxonomy of Colias hecla species group is obscure (Gorbunov 2001), and therefore we consider the records of $C$. aquilonaris Gr.Grsh. from Maymecha r. [Korshunov \& Gorbunov (1995), as Colias viluiensis; Korshunov $(1998,2000)]$ as belonging to $C$. hecla. HA.

\section{Lycaenidae}

[Lycaena helle (Den. et Schiff.)] - This species was erroneously mentioned from Taymyr by Korshunov et al. (1982); however, the northernmost record of this species [Yenisey valley, $65^{\circ} 35^{\prime} \mathrm{N}$; Trybom (1878)] is far from Taymyr.

Lycaena phlaeas (L.) - Talnakh: $1 ð$ 5.VIII.1964 [Korshunov et al. (1982), as ssp. hypophlaeas Bsd.]. HA.

Vacciniina optilete (Knoch.) - Popigay r., low flow: $1 ð$ 16.VII.1967, 10 1.VIII.1968 (Korshunov et al. 1982); Nosok: $5 \AA$ 1 21.28.VII.1977 (Korshunov et al. 1982); Dudinka: 25.VII.1876 [Trybom (1878), as ssp. cyparissus Hb.], 50 đ̄ 2 \% 1.-12.VII.1915 (W), $1 ð$ 23.VII.2002 (K); Vershininskoe: 26.VII.1876 [Trybom (1878), as ssp. cyparissus $\mathrm{Hb}$.]; Kayyerkan: 1 \% 26.VII.1970 (Korshunov et al. 1982); Noril'sk: $2 \widehat{\jmath} 2$ ㅇ 21.-25.VII.2002 (K); Talnakh: $1 \delta^{\Uparrow} 2$ ㅇ 29.VII.2002 (K), $2 \hat{\jmath}$ 14.VII.2001 (G), $3 \curvearrowright 1$. \& 5.VIII.1964 (Korshunov et al. 1982); Eremin's hut: $7 \lesssim 5 \bigcirc$ 28.VII.2002 (K); Lama: 3 ㅇ 26.-28.VII.2002 (K); Potapovo: 25.VII. 1876 [Trybom (1878), as ssp. cyparissus

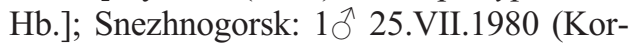
shunov et al. 1982); Rybnaya r.: $7 \overbrace{}^{\lambda}$ 12.23.VII.1978 (Korshunov et al. 1982); Keta lake: 1ठ઼ 22.VII.1980 (Korshunov et al. 1982); Khantayskoe lake: $1 \hat{\jmath}$ VIII.1980 (Korshunov et al. 1982). Korshunov et al. (1982) classified all records from Taymyr as ssp. cyparissus Hb. HA.

Polyommatus icarus (L.) - Talnakh: $3 ð 2$ đ 4.5.VIII.1964 (Korshunov et al. 1982). PA.

Polyommatus kamtschadalis (Shelj.) - 'Taymyr': Antonova (1976), as P. eros; Talnakh: $1{ }^{\lambda} 15 . V I I .2001(\mathrm{G}), 1{ }^{\curvearrowright} 1$ 우 29.VII.2002 (K), $4{ }^{\lambda} 1$ ㅇ 4.-5.VIII.1964 (Korshunov et al. 1985); Rybnaya r.: $10 \overbrace{}^{\lambda} 2$ 27.VII.1978 [Korshunov et al. (1982), as P. eros taimyrensis Korsh.]; Keta lake: $2 \widehat{\jmath} 22$.VII. 1980, 1ठ̄ 28.VII.1980 [Korshunov et al. (1982), as P. eros taimyrensis Korsh.]. Subspecies taimyrensis was transferred to $P$. kamtschadalus by Korshunov \& Gorbunov (1995). EP.

Agriades glandon wosnesenskii Mén. - Popigay r.: 1 + 19.VII.1967, 1 9 30.VII.1968 [Korshunov et al. (1982), as A. aquilo wosnesenskii Mén.]; Chopko r.: 1ㅇ 12.VII.1976 [Korshunov et al. (1982), as A. aquilo wosnesenskii Mén.]; Noril'sk: 3 21.-25.VII.2002 (K); Talnakh: 20 14.VII.2001 (G). HA.

Nymphalidae

Nymphalis xanthomelas (Esper) - Verkhnyaya Taymyra r.: $1{ }^{\lambda} 17$. VIII.1983 (Korshunov et al. 1985); Dudinka: 1 ex. 1.-12.VII.1915 (W); Talnakh: 5.-6.VIII.2001 (G); Eremin's 
hut: 1 + 28.VII.2002 (K); Rybnaya r.: 1 ㅇ 12.VII.1976 (Korshunov et al. 1982). PA.

Vanessa cardui (L.) - Tareya (Chernov 1978); Rybnaya r.: 1ठ̂ 12.VII.1978 (Korshunov et al. 1982). Cosmopolitan.

Euphydryas iduna (Dalm.) - Dudinka: 19 11.VII.1977 (Korshunov et al. 1982); Kayyerkan: 1ð 26.VII.1970 (Korshunov et al. 1982); Rybnaya r.: 1 đ 23.VII.1978 (Korshunov et al. 1982). PA.

Boloria eunomia (Esper) - Tareya: $2 \hat{\sigma} 2$ ㅇ 31.VII.-3.VIII.1975 (Korshunov et al. 1985); Popigay r., low flow: $2 \widehat{\delta} 1 q$ 16.-19.VII. 1967, 1ठ 30.VII.1968 (Korshunov et al. 1982); Ary-Mas: 2 29.VII.1981, $2 \AA$ VII.1983 (Korshunov et al. 1985); Boganida r. [Ménétriès (1851), as Argynnis aphirape]; Chopko r.: 1 đ̊ 12.VII.1976 (Korshunov et al. 1982); Zaostrovskaya: 3 exx. 1915 (W); Nosok: 8 đิ 8 ㅇ 17.-22.VII.1977 (Korshunov et al. 1982); Dudinka: 13 exx. 1.-12.VII.1915 (W), 10 11.VII.1977 (Korshunov et al. 1982); Noril'sk: 1 đิ 6.VII. 1977 (Korshunov et al. 1982); Talnakh: $1{ }^{\lambda} 1$ q 23.VII.1964, $1{ }^{\lambda}$ 1 q 29.VII.1964, 1ठ 31.VII.1964, $1 \overbrace{}^{\lambda}$ 4.VIII.1964 (Korshunov et al. 1964), $2 \hat{\jmath} 16 .-$ 17.VII.2001 (G); Rybnaya r.: 1ठ 4.VII.1976, 24ð 3 12.-22.VII.1978 (Korshunov et al. 1982); Ketairbe r.: $1 \bigcirc 1 \bigcirc 15 . V I I .1982$ (Korshunov et al. 1985); Kutaramakan r.: $1 \sigma^{\hat{\gamma}}$ 12.VIII.1980 (Korshunov et al. 1982); Khantayka: 15.VII.1876 [Trybom (1878), as ssp. ossianus Herbst]. HA.

[Boloria euphrosyne L.] - Dudinka: 29.VII.1876 (Trybom 1878). This is the only record of this species from Taymyr. Trybom (1878) mentioned that the specimens are old and worn, and that therefore it is impossible to be sure that they belong to this species. Korshunov et al. (1982) considered this record doubtful.

Boloria selene (Den. \& Schiff.) - Khantayka: 20.VII.1876 [Trybom (1878), as ssp. hela Stgr.]; Snezhnogorsk: $3 \hat{\jmath}$ 22.-25.VII.1980 [Korshunov et al. (1982), as ssp. thalis $\mathrm{Hb}]$. PA.

Boloria frigga (Thunbg.) - Tareya: $1 \uparrow 18$.VII. 1969, 1 ㅇ 24.VII.1969 (Chernov 1973, 1978; Korshunov et al. 1982), $6 \curvearrowright 17$ 우 1975 (Korshunov et al. 1985); Popigay r., low flow: $3{ }^{\wedge}$ 17. \& 19.VII.1967, 1 + 23.VII.1968 (Korshu- nov et al. 1982); Ary-Mas: $2 \precsim 2$ ○ VII.1983 (Korshunov et al. 1985); Boganida r. (Ménétriès 1851); Dudinka: 25.VII.1876 (Trybom 1878), 1 ex. 1.-12.VII.1915 (W), 1 1 11.VII.1977 (Korshunov et al. 1982); Rybnaya r.: 1 đิ 5.VII.1978 (Korshunov et al. 1982); Khantayka: 19.VII.1876 (Trybom 1878). HA.

Boloria improba (Butl.) - Tareya: infrequent in 1975 (Korshunov et al. 1985); Ary-Mas: 1 ex. VII.1983 (Korshunov et al. 1985). HA.

Boloria thore borealis (Stgr.) - Chopko r.: $1 \widehat{\jmath}$ 10.VII.1976 (Korshunov et al. 1982); Talnakh: $1 \delta$ 15.VI.1964, $1 \delta 3 \uparrow$ 4.-6.VIII. 1964, 1ð 31.VII.1964 (Korshunov et al.

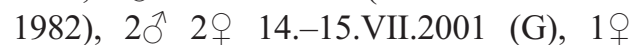
29.VII.2002 (K); Eremin's hut: $1 \sigma^{\hat{\prime}} 1$ 우 28.VII.2002 (K); Lama: 1ð 26.-28.VII.2002 (K); Snezhnogorsk: $9 ð 4 \uparrow$ 22.-25.VII.1980 (Korshunov et al. 1982); Potapovo: 23.VII. 1876 (Trybom 1878); Rybnaya r.: $1 \hat{\jmath}$ 18.VII.1976, 1ठึ 19.VII.1978 (Korshunov et al. 1982); Kutaramakan r.: 1 đ 12.VIII.1980 (Korshunov et al. 1982); Khantayka: 19.VII. 1876 (Trybom 1878), 1 ㅇ 29.VII.1965 (Korshunov et al. 1982). HA.

Boloria angarensis (Erschoff) - Ary-Mas: $1 \hat{\sigma}$ VII.1983 (Korshunov et al. 1985); Chopko r.: 1 ㅇ 5.VII.1976, 1 ㅇ 14.VII.1976 (Korshunov et al. 1982); Nosok: 1ð 28.VII.1977 (Korshunov et al. 1982); Talnakh: 1 त $1 q$ 23.24.VII.1964, 1 q 26.VII.1964, 1 \% 5.VIII. 1964 (Korshunov et al. 1982), $4 \lesssim 1$ 우 13.20.VII.2001 (G); Eremin's hut: $2 \hat{\jmath} 2$ ㅇ 28.VII.2002 (K); Rybnaya r.: $1 \delta^{\hat{~}} 18$.VII. 1976, 1 § 3 ㅇ 15.-22.VII.1978 (Korshunov et al. 1982); Kutaramakan r., middle flow: $1 \sigma^{\lambda}$ 12.VIII.1980 (Korshunov et al. 1982). EP.

Boloria selenis (Ev.) - Talnakh: 6ð 13.17.VII.2001 (G). EP.

Boloria chariclea (Schneider) - Tareya: $3 \lambda 1 q$ 30.VII.-4.VIII.1975 [Korshunov et al. (1985), as ssp. arctica Zett.]; Talnakh: $1 \delta^{\lambda}$ 1.VIII.1964 [Korshunov et al. (1982), as ssp. boisduvali [sic!] Dup.]; Khantayka: 19.VII. 1876 [Trybom (1878), as aber. boisduvalii Dup.]. HA.

Boloria tritonia machati Korsh. - 'Taymyr': Antonova (1976), as Clossiana erda Chr.; Talnakh: 1ð 11.VII.1964 [Korshunov et al. 
(1982), as C. erda Chr.], $2 \hat{\jmath} 4$ ㅇ 13.20.VII.2001 (G) (determined as C. distincta machati Korsh. by B. Khramov); Ketairbe r.: 1ठ 18.VII.1981 [Korshunov et al. (1985), as C. distincta Gibs.; Korshunov (1987), as C. distincta machati; Gorbunov (2001), as Boloria tritonia machati]. Although erda $\mathrm{Chr}$. is the junior synonym of polaris Boisd. (Gorbunov 2001), we attribute the earlier records of erda to B. tritonia. BE.

Boloria polaris (Boisd.) - Meduza Bay (Syroechkovskij et al. 2000); Portnyagino lake: $3{ }^{\lambda}$ 8.-10.VIII.1933; Yamutarida r.: $7 \lesssim$ 6 6.-16.VII.1928 (Tolmachev); Verkhnyaya Taymyra r.: $1 \delta$ 17.VII.1983 (Korshunov et al. 1985); Tareya: Chernov (1978), 2ð 9.VII.1969 (Korshunov et al. 1982), 41 ${ }^{\lambda}$ 32 ㅇ VII.-VIII.1974/1975 (Korshunov et al. 1985); Gorbita r.: 1ð 14.VII.1929 (Makarjin); Popigay r., middle flow: 19 8.VII.1968

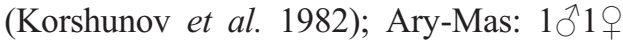
VII.1983 (Korshunov et al. 1985); Boganida r. (Ménétriès 1851); Shaitan mt. (old name), $72^{\circ} \mathrm{N}$ (?) (Lewaski 1887); Byrranga mts., $75^{\circ}$ N (Lewaski 1887); Nosok: 10َ 17.VII.1977 (Korshunov et al. 1982); Dudinka: $1 \hat{\sigma}$ 22.VII.1928 (Tolstoj). HA.

Boloria freija (Thnbg.) - Tareya: $4 \bigcirc 3 q$ 28.VII.3.VIII.1975 (Korshunov et al. 1985); AryMas: 3 ^ै VII.1983 (Korshunov et al. 1985); Khatanga: $2 \hat{\jmath} 4+$ 2.-15.VII.1905 (Tolmachev); Mouth of Yenisey r.: 1 ex. 1901 (S); Dudinka: $1{ }^{\Uparrow}$ 26.VII.1907 (Tolstoj), 1 ex. 1.12.VII.1915 (W), 1ð 30.VI.1977 (Korshunov et al. 1982); Kayyerkan: 1 đ̊ 26.VII.1970 (Korshunov et al. 1982); Talnakh: 10 17.VII.1964, 2 q 26.VII.1964 (Korshunov et al. 1982), 1ð 13.VII.2001 (G); Fokina r.: $11_{1}$ q early VII.1978 (Korshunov et al. 1982); Rybnaya r.: 12 ð’9ㅇ 12.-22.VII.1978 (Korshunov et al. 1982); Ketairbe r.: 1 ふ 12.VII.1982 (Korshunov et al. 1985); Khantayka: 15.VII.1786 (Trybom 1876), 1 ex. 1901 (S); Monjero, system of Khatanga $\mathrm{r}$. (?): 12 đ V.-VI.1874 (Chekanovskij). HA.

Boloria alaskensis Hld. - Verkhnyaya Taymyra r.: $1{ }^{\wedge}$ VII.1983 (Korshunov et al. 1985); Tareya: 10 ${ }^{\lambda}$ 4 1 18.-24.VII.1969 [Korshunov et al. (1982), as Boloria aquilonaris

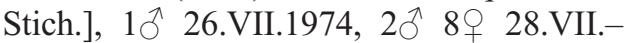

3.VIII.1975 (Korshunov et al. 1985); Kunzhalak lake: 1 + 21.VII.1967 [Korshunov et al. (1982), as Boloria aquilonaris Stich.];

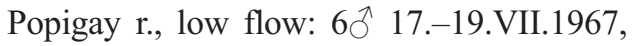
$2 \hat{\jmath} 2$ ㅇ 14.-30.VII.1968 [Korshunov et al. (1982), as Boloria aquilonaris Stich.]; AryMas: 1ठ 29.VII.1981 (Korshunov et al. 1985); Zaostrovskaya: $3{ }^{\wedge} 1915$ (W); Dudinka: $1 \delta$ 1우 1.-12.VII.1915 (W); Talnakh: $25 \hat{\jmath}$ 10ㅇ 14.-20.VII.2001 (G); Khantayka: 1 ㅇ 1901 (S). Most likely the record of 'Boloria pales' from Tareya (Chernov 1973) belongs to this species. BE.

Boloria aquilonaris (Stich.) - Chopko r.: 2今 12.14.VII.1976 (Korshunov et al. 1982); Nosok:

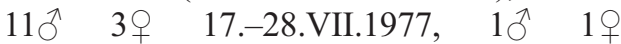
25.VII.1977 (Korshunov et al. 1982); Rybnaja r.: 13ठ̂ 18.-27.VII.1978 (Korshunov et al. 1982). PA.

Issoria eugenia (Ev.) - Dudinka: 1 ex. 3.VII.1876 (Trybom 1878); Noril'sk: $2 \bigcirc 2 \bigcirc$ 6.VIII.1964 (Korshunov et al. 1982); Talnakh: 1 đ̊ 1오.3.VIII.1964 (Korshunov et al. 1982), $8{ }^{\lambda} 3$ 우 17.VII.2001 (G). EP.

Satyridae

Coenonympha tullia (Müll.) - Noril'sk: $1 \delta^{\Uparrow} 2$ ㅇ 21.-25.VII.2002 (K), 1 q 30.VII.200r (K); Snezhnogorsk: 2 त 25.VII.1980 [Korshunov et al. (1982), as ssp. viluiensis Mén.]. HA.

Erebia jeniseiensis Trybom - Talnakh: 3ð 23.26.VII.1964, 1 9 5.VIII.1964 (Korshunov et

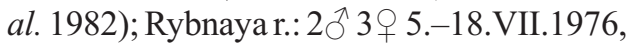
$43{ }^{\Uparrow} 9$ ㅇ 12.-27.VII.1978 (Korshunov et al. 1982); Khantayka: 16.-19.VII.1876 [Trybom (1878), as ligea var. jeniseiensis]; Snezhnogorsk: 1ð̋ 1ㅇ 22.-25.VII.1980 (Korshunov et al. 1982). EP.

Erebia rossii Curt. - Tareya: $1 ð$ 25.VII.1974, 2ð 1.VIII.1975 [Korshunov et al. (1985), as ssp. ero Brem.]; Ary-Mas, Novaya r.: 2ठ VII.1981 (P) [Korshunov (1996), as ssp. subarctica Korsh.; Korshunov (2002), as ssp. yamala Korsh.]; Chopko r.: 20 8.VII.1976 [Korshunov et al. (1982); Korshunov (1996), as ssp. subarctica Korsh.; Korshunov (2002), as ssp. yamala Korsh.]; Maymecha r.: 105 ㅇ 28.VII.-8.VIII.1982 (Zakharzhevskii) [Korshunov (1996), as ssp. subarctica Korsh.; Korshunov (2002), as ssp. yamala Korsh.]; 


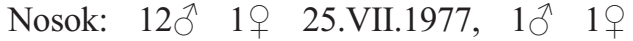
22.VII.1977 (Korshunov et al. 1982); Dudinka: 9 exx. 1.-12.VII.1915 (W); Tal-

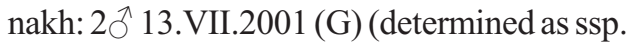
kuskoquima Holland by B. Khramov); Khantayka: 19.VII.1876 [Trybom (1878), as E. ero]. BE.

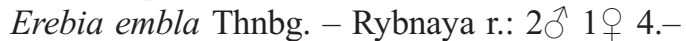
5.VII.1976, 2ð 1ㅇ 18.-21.VII.1978 (Korshunov et al. 1982); Khantayka: 15.-18.VII. 1876 (Trybom 1878). PA.

Erebia disa (Thnbg.) - 'from Vorkuta to East Taymyr' (Chernov 1966); Ary-Mas: $1 \delta$ VII.1983 (Korshunov et al. 1985); Chopko r.: 1今 4.VII.1976 (Korshunov et al. 1982); Dudinka: 13 exx. 1.-12.VII.1915, (W); Kayyerkan: 1 đ 26.VII.1970 (Korshunov et al. 1982); Noril'sk: 1 q 6.VII.1977 (Korshunov et al. 1982); Talnakh: 1ठ̄ 11.VII.1964, 1ठิ 17.VII.1964 (Korshunov et al. 1982); Ketairbe r.: $7 ð 18$.VII.1982 (Korshunov et al. 1985); Khantayka: 19.VII.1876 (Trybom 1878). HA.

Erebia fasciata semo Gr.-Gr. - Verkhnyaya Taymyra r.: 1ठ 20.VII.1983 (Korshunov et al. 1985); Tareya: Chernov (1973, 1978), 56ð 115우 VII.-VIII.1974/1975 (Korshunov et al. 1985); Popigay r., low flow: $1 \overbrace{}^{\Uparrow} 3$.VII. 1967 (Korshunov et al. 1982); Dudinka: 1 ex. 1.-12.VII.1915 (W). Had long been considered as a separate species, E. semo (Korshunov \& Gorbunov 1995, Korshunov 1996, 1998, 2000, 2002); however Gorbunov (2001) classified semo as subspecies of $E$. fasciata. BE.

Erebia discoidalis (Kirby) - Chopko r.: $1{ }^{\lambda}$ 8.VII.1976 (Korshunov et al. 1982); Khantayka: 19.VII.1876(Trybom 1878). BE.

Erebia dabanensis Erschoff - Chopko r.: $1{ }^{\AA}$ 17.VII.1976 (Korshunov et al. 1982); Talnakh: $1 \hat{\jmath} 1$ ㅇ 15.VII.2001 (G). BE.

Oeneis melissa also (Boisd.) - Tareya: $2{ }^{\lambda} 3$ ㅇ

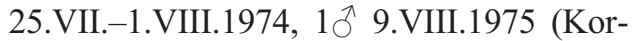
shunov et al. 1985); Talnakh: 1 q 30.VII.1985 (M), 2 \% 27.VII.1999 (M), $16 \jmath^{\Uparrow} 3$ 13.17.VII.2001 (G) (determined as subsp. karae Kusn. by B. Khramov); Ketairbe r.: $2 \hat{\jmath} 1$ ㅇ 18.VIII.1982 (Korshunov et al. 1985). BE.

[Oeneis jutta Hb.] - Khantayka: 19.VII.1876 (Trybom 1878). This is the only record of this species from Taymyr. Korshunov et al. (1982) mentioned this record, but in the next publication (Korshunov et al. 1995) no data on $O$. jutta were included for Taymyr.

Oeneis magna Graes. - Talnakh: 1 9 30.VII.1985 (M) (determined as ssp. pupavkini Korsh. et Gorb. by B. Khramov); Rybnaya r.: 2 + 7.12.VII.1976, 11§ึ 1ㅇ 12.-27.VII.1978 (Korshunov et al. 1982; Korshunov \& Gorbunov 1995). Korshunov (2002) refers to Oeneis pupavkini and Oeneis magna magadanica from Taymyr. EP.

Oeneis crambis Frr. - Tareya: 1 đ VII.1969 (Korshunov et al. 1985); Ary-Mas: 1 + VII.1981, 1 ㅇ VII.1983 [Korshunov et al. (1985); Korshunov (2002), as O. putorana Korsh. et Nikol.]; Nosok: 21.VII.1977 (P) [Korshunov (2002), as O. putorana Korsh. et Nikol.]; Maymecha r., upper flow: 19 21.VII.1982, 1 ㅇ 26.VII.1982, 2 ㅇ 2.VIII.1982 (Zakharzhevskii) [Korshunov (2002), as O. putorana Korsh. et Nikol.]. EP.

Oeneis bore (Schn.) - Tareya: 1 + 3.VIII.1975, 1 ㅇ 4.VIII.1985 (Korshunov et al. 1985); Dudinka: 1 ex. 1.-12.VII.1915 (W); Talnakh: $1{ }^{\nearrow}$ 30.VII.1985 (M) (determined as $\mathrm{f}$. ammonoides Tatarinov et Dolgin by $\mathrm{B}$. Khramov); Rybnaya r.: 1ठ 13.VII.1978 (Korshunov et al. 1982); Khantayka: 15.18.VII.1876 [Trybom (1878), as var. taygete Hb.], 1 ㅇ 1901 (S). HA.

\section{Geometridae}

Scopula frigidaria (Möschler) - Dika Bay: $3 \hat{\jmath}$ 21.VI.-4.VII.1915 (Starokadomskij); Taymyr Island (missing on Fig. $1 ; 76^{\circ} 22^{\prime} \mathrm{N}$,

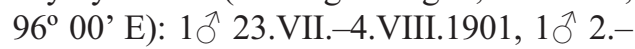
15.VIII.1901, 1ठ 10.-23.VII.1909 (Birulja); Portnyagino: 1ठ 8.-10.VIII.1933 (Yakovlev); Zaostrowskaya: 1ô 1 † 1901 (W); Dudinka: $2 \hat{\jmath}$ 2q, 1.-12.VII.1901 (W); Noril'sk: 4 `3우 21.-29.VII.2001 (K); Talnakh: 3 đ 29.VII. 2002 (K); Eremin's hut: $3 \hat{\jmath}$ 28.VII.2002 (K); Lama: 5ô 1 ㅇ 26.28.VII.2002 (K); Sobach'e lake: $1 \delta^{\hat{\sigma}}$ 22.VII.1996 (M\&B); Gusikha r.: $1 \sigma^{\hat{\sigma}}$ 27.VII.1933 (Yakovlev). HA.

Xanthorhoe decoloraria (Esper) - Noril'sk: $6 \AA$ 1 ㅇ 21.-25.VII.2002 (K); Talnakh: 2 ㅇ 29.VII.2002 (K). HA. 
Xanthorhoe ferrugata (Cl.) - Noril'sk: $3 \hat{\jmath} 2 q$ 21.-25.VII.2001 (K). HA.

Xanthorhoe derzhavini (Djak.) - Ary-Mas: 1 + VII.1982 (Vasilenko 1995). Nizhnyaya Agapa r.: $1 \delta^{\wedge}$ 20.VII.1999 (M\&B). The latter specimen may represent subsp. jakuta Vasilenko, although the taxonomic affinities of this subspecies remain unclear (V. Mironov, pers. comm.). EP.

Spargania luctuata (Den. et Schiff.) - Noril'sk: 1`3 9 21.-25.VII.2001 (K); Talnakh: 1 ㅇ 29.VII.2002 (K). HA.

Rheumaptera hastata (L.) - Cheluskin cape: 1 ex. 2.VII.1934 (parts of wings only); Lama: $2{ }^{\lambda}$ 1 ㅇ 26.-28.VII.2002 (K); Eremin's hut: 7ð 2 2 28.VII.2002 (K). HA.

Rheumaptera subhastata (Nolcken) - Noril'sk: 1ठ 21.-25.VII.2001 (K); Talnakh: 2 ㅇ 29.VII.2002 (K); Eremin's hut: 7ठ 5우 28.VII.2002 (K); Lama: $1 \delta^{\hat{\gamma}} 3$ ㅇ 26.28.VII.2002 (K); Sobach'e lake: 1 + 22.VII. 1996 (M\&B). HA.

Rheumaptera undulata (L.) - Eremin's hut: 1 + 28.VII.2002 (K). HA.

Psychophora cinderella Viidalepp - N. Taymyr (7604'30' N, 98²' W): 2ð 12.-17.VII. 1991 (Hildén); paratypes: Viidalepp (2001). EP.

Psychophora sabini (Kirby) - Meduza Bay: $1 \hat{\sigma}$ 2 + 10.-15.VII.1998(Kh); Sobach'e lake: $1{ }^{\top}$ 22.VII.-13.VIII.1996 (M\&B). HA.

Entephria punctipes (Curt.) - Ary-Mas: 403 ㅇ 27.VI.-16.VIII.1983 (Vasilenko 1990). HA.

Entephria polata (Dup.) - Zaostrowskaya: $1{ }^{\AA}$ 4ㅇ 1901 (W); Dudinka: 1ठ̋ 1.-12.VII.1901 (W). It is likely that the record of 'Cidaria polita Dkp.' [sic!] from Tareya (Chernov 1973) concerns this species. HA.

Aspitates orciferarius Wlk. - Dudinka: 19 1.12.VII.1915 (W). BE.

Aspitates taylori sibirica Djak. - Khantayka: 10 1 19 19.VII.1901 (S); Khatanga: 1ठ 1.14.VII.1905 (Tolmachev); Dudinka: 1 ภ 2 ㅇ 1.-12.VII.1915 (W). BE.

Aspitates sp. - Tareya [Chernov (1973), mentioned as 'Aspilates sp.'].

Lycia lapponaria (Boisd.) - Monjero r., $\begin{array}{llll}\text { Khatanga system (?): } 1 ठ & \text { V.1874 }\end{array}$ (Tshekanovskij). PA.
Sphingidae

Hyles gallii (Rott.). - Noril'sk: 1ð 21.-25.VII. 2002 (K). Numerous larvae have been collected from Epilobium angustifolium in the city and on wastelands around it, and several specimens emerged by 5.VIII.2002. HA.

Lymantriidae

Gynaephora rossii (Curt.) - N. Taymyr $\left(76^{\circ} 04^{\prime} 30^{\prime \prime} \mathrm{N}, 98^{\circ} 32^{\prime} \mathrm{E}\right)$ : $8{ }^{\jmath} 5$ ㅇ 12.17.VII.1991 (Hildén); Maymecha r., upper flow, $700 \mathrm{~m}$ a.s.1.: 1ㅇ, 1 larva 19.VII.1982 (Zakharzhevskii); Tareya (Chernov 1973); Ary-Mas: 19 5.VIII.1981 (P). BE.

Arctiidae

Dodia albertae eudiopta Tshist. - $25 \mathrm{~km} \mathrm{~S}$ of Kayak: $5 \hat{\jmath} 2$ (Savenkov); Talnakh: $1 \widehat{\jmath}$ 23.VII.1964 [both records mentioned by Dubatolov \& Zolotarenko (1990) and Dubatolov et al. (1997)]. BE.

Acerbia alpina (Quens.) - Bol'shaya Balakhnya r.: 1 q 25.VII.1934 (Yakovlev) [Sotavalta (1962, 1963) cited this record as River Balakhi; also mentioned by Dubatolov et al. (1997)]; Ary-Mas: 1 đ 3.VIII.1981 (P). HA.

Pararctia lapponica Thnbg. - 'Taymyr': Sotavalta (1965), Dubatolov \& Zolotarenko (1990); Tareya: $1 \precsim 5$.VIII.1974 (Elshin) [record mentioned by Dubatolov et al. (1997) as 'Gnareja' - lapsus calami], 1 1 13.VII.1966, 1ð 17.VII.1966, 1 ` (ех рupa) VII.1966 (C); Khatanga: $1 \sigma^{\widehat{A}}$ (ex pupa) 22.VI.1985 (Detkova). HA.

Pararctia subnebulosa tundrana Tshist. Svyatovo Pavla [Is.] (old name): Tshistjakov (1990), as P. tundrana; mouth of Yenisey $75^{\circ}$ $\mathrm{N}, 85^{\circ} \mathrm{E}$ (in fact, these coordinates refer to sea far from Yenisey): $1 \widehat{\jmath} 1914$ [Sotavalta (1965), as Hyphoraria subnebulosa Dyar]; Tareya: $1 \delta^{\Uparrow}$ VII.1967 (C) [Chernov 1973, as Hyphoraia subnebulosa Dyar]; Ary-Mas: 10 5.VIII.1981 (P); Kresty: 1 đิ 1 + 12.VII.1976 (Eskov); Malaya Lagota r., left tributary of Bolshaya Lagota r., right tributary of East Taymyra r.: $1 \overbrace{}^{\lambda}$ VII.1984 (Chupin); Nizhnyaya Agapa r., $40 \mathrm{~km}$ from the headwater: 1ठ 23.VII.1973 (Zherikhin); Noril'sk: 1ठ 13.-18.VII.1967 [Tshistjakov (1990), as P. tundrana]. EP. 
Eilema vakulenkoi Tshist. - Ary-Mas: $1 ð 15$.VII. 1985 (Chupin) (Dubatolov \& Zolotarenko 1990). EP.

\section{Noctuidae}

[Euchalcia modestoides Poole] - 'Taymyr' [Kljuchko (1985), as E. modesta Hb.]. The record is doubtful as the northern distribution range of this species lies much more south (see Goater et al. 2003).

Syngrapha diasema (Boisd.) - Dudinka (Kljuchko 1988); Eremin's hut: 1ठ઼ 28.VII. 2002 (K). HA.

Syngrapha interrogationis (L.) - Dudinka (Kljuchko 1988); Eremin's hut: $1 \circlearrowleft$ 28.VII. 2002 (K). HA.

Syngrapha transbaikalensis Stgr. - Dudinka (Kljuchko 1988). EP.

Syngrapha hochenwarthi (Hochw.) - Dudinka: $1 \hat{\sigma}^{\hat{N}}$ 23.VII.1955 [record mentioned by Kljuchko (1988) and Zolotarenko (1990) as Caloplusia hochenwarthi]. PA.

Syngrapha parilis (Hb.) - 'Taymyr': Kononenko (1997). HA.

Sympistis heliophila (Payk.) - Talnakh: 10 29.VII.1964, 1ठ 4.VIII.1964 (Z) (Zolotarenko 1990); Eremin's hut: $5 \hat{\jmath}$ 28.VII.2002 (K); Khantayskoe lake: $1{ }^{\lambda}$ 15.VII.1980 (P) (Zolotarenko 1990). HA.

Sympistis nigrita (Boisd.) - Sobach'e lake: 10`2市 22.VII.-13.VIII.1996 (M\&B); 'Taymyr': Chernov (1973, 1978), Kononenko (1997). All records belong to ssp. zetterstedti Stgr.]. HA.

Sympistis lapponica (Thnbg.) - $25 \mathrm{~km} \mathrm{~S}$ of Kayak: 2 exx. 29.VII.1980 (Savenkov) (Kononenko 1997); Sobach'e lake: 1 q 13. VIII. 1996 (M\&B). HA.

Hillia iris (Zett.) - Snezhnogorsk: 1ð 10.VIII. 1965 (Kharinova) (Zolotarenko 1990). HA.

Coranarta carbonaria (Chr.) - 'Taymyr': Kononenko (1997); unidentified locality between Noril'sk and Dudinka [indicated in figure 25 by Lafontaine et al. (1987b)]. EP.

Papestra biren (Goeze). - Lama: $1 \precsim 26 .-28 . V I I$. 2002 (K). HA.

Polia richardsoni Zett. - Tareya: 1 ex. VII.1966 (C) (Chernov 1973, 1978, Kononenko 1997); Nizhnyaya Agapa r.: 1 ex. 23.VII.1973 (Zherikhin) (Kononenko 1997); Ami-Neri
(?): 1 ๙ 12.VIII.1928 (Tolmachev). HA.

Lasionycta staudingeri (Möschler) - Khatanga [locality indicated in figure 60 by Lafontaine et al. (1986)]; Kresty: 1 ex. 31.VII.1982 (Zherikhin) (Kononenko 1997); Talnakh: $1 \widehat{\jmath}$ 30.VIII.1964 [Zolotarenko (1970, 1990); in the last paper the collection date is given as 1.VIII.1964]. HA.

Lasionycta leucocycla albertensis (McDonnough) - Bol'shaya Romanikha r., $11 \mathrm{~km}$ from mouth: 2 exx. 11.VII.1977 (Zherikhin) (Kononenko 1997); Maimera r., 3 km from mouth 25.VII.1971 (Ponomarenko) (Kononenko 1997); Bol'shaya Kheta r. near Dudinka: 1 స 28.VII.1966; $80 \mathrm{~km} \mathrm{~S} \mathrm{Khatanga}$ [indicated in figure 59 by Lafontaine et al. (1986)]. HA.

Xestia liquidaria (Ev.) - $25 \mathrm{~km} \mathrm{~N}$ of r. Gusikha mouth: 2 27. VII.1933 (Yakovlev); Meduza Bay (Syroechkovskij et al. 2000); unidentified locality at $76^{\circ} \mathrm{N}, 110^{\circ} \mathrm{E}$ [figure 9 in Lafontaine et al. (1983)]; Tareya: 1 ex. 9.VII.1966 (C) (Chernov 1973, 1978, Kononenko 1997); Gorbita r.: $9{ }^{\lambda}$ 20.VII.1929 (Makarjin); Ary-Mas: 20ิ VII.1983 (P); Boganida r.: Ménétriés (1851), as Amphidasis unifasciata Men.; Uboinaya r.: 30.-31.VII.1984 (Tomkovich) (Kononenko 1997); Malaya Lagota r. mouth: $1 \delta$ 29.VII.1983 (P). BE.

$X$. aequaeva (Benjamin) - 'Taymyr': 20 12.VII.1946 (Svizhenko); Taymyr Island (missing on Fig. 1; $\left.76^{\circ} 22^{\prime} \mathrm{N}, 96^{\circ} 00^{\prime} \mathrm{E}\right)$ : 1 ex. 26.VII.-10.VIII.1901 (Birulja) (Kononenko 1997); N. Taymyr (7604'30' N, 98³2' E): 2 ㅇ 12.-17.VII.1991 (Hildén); unidentified locality at $76^{\circ} \mathrm{N}, 110^{\circ} \mathrm{E}$ [figure 4 in Lafontaine et al. (1983)]; Tareya: 1 ex. VII.1966 (C) (Kononenko 1997); Uboinaya r.: 25.VII.1984 (Tomkovich) (Kononenko 1997). BE.

X. speciosa (Hb.) - Talnakh: 5ð 4.-6.VIII.1964 (Z) (Zolotarenko 1970, 1990). HA.

$X$. laetabilis (Zett.) - Talnakh: 20̄ 22.VII.1964 (Z) (Zolotarenko 1970, 1990); Noril'sk: 20 4.VIII.1964 (Z) (Zolotarenko 1970). PA.

X. tecta (Hb.) - 'Taymyr': Kononenko (1997); unidentified locality near Taymyr lake [figure 1 in Lafontaine et al. (1987a)]; Ary-Mas: 1 ㅇ 3.VIII.1981 (P) (Zolotarenko 1990); Talnakh: 
1 우 (ex pupa) 20.VII.1964, $20 \hat{~ 26 . V I I .-~}$ 4.VIII.1964 (Z) (Zolotarenko 1970, 1990). HA.

$X$. okakensis morandi (Benjamin) - Dudinka: 1.VIII.1878 (S) [Lafontaine et al. (1987a), as X. okakensis okakensis Packard]. BE.

$X$. lorezi kongsvoldensis (Grönlien) - Unidentified locality between Noril'sk and Dudinka [indicated in figure 4 by Lafontaine et al. (1987a)]; Talnakh: 1 ex. 6.VIII.1964 [Zolotarenko (1990), as X. sajana, now considered ssp. of lorezi]. HA.

$X$. fergusoni Lafontaine - Kresty: 19 12.VII. 1976 (Eskov) (Kononenko 1997). BE.

X. quieta (Hb.) - Ary-Mas: 1ठ 5.VIII.1981 (P); $25 \mathrm{~km} \mathrm{~S}$ of Kayak: 2 exx. 19.-27.VII.1980 (Savenkov) (Kononenko 1997); Ayan lake: 1 ex. 15.VII.1983 (Eskov) (Kononenko 1997); Khantayka: 2ð 15.VII.1876 (Jacobson) (Kononenko 1997); Bai-Mura-Nery Bay (?): 1 ex. 28.VII.1976 (Kononenko 1997). HA.

X. albuncula (Ev.) - Noril'sk: Zolotarenko (1970), as X. hyperborea Zett.; Talnakh: 90 2 7 25.VII.-6.VIII.1964 (Z) [Zolotarenko (1990) mentioned 74 exx. from Talnakh, possibly counting also unpinned materials]. BE.

X. penthima (Erschoff) - 'Taymyr' (Kononenko 1997). EP.

$X$. innuitica Laf. \& Hansel. - Talnakh (Kononenko 2005). BE.

Actebia fennica (Tausch.) - Noril'sk (Zolotarenko 1970). HA.

Trichosilia arctica Kononenko - Ary-Mas: 1ठ 5.VIII.1983 (P) [Zolotarenko (1990), as T. arctia]. BE.

Euxoa ochrogaster (Gn.) - Noril'sk [Zolotarenko (1970), as E. islandica Stgr.]. HA.

Euxoa churchillensis (McDunnough) - Tareya:

2ð 1 우 (ex larva) 3.-5.VII.1968 (C)

(Kononenko et al. 1996). BE.

\section{Discussion}

The distribution of moths and butterflies at the extreme North of Eurasia is documented quite poorly. Among the regions beyond the Polar Circle, comprehensive faunistic lists (including all taxa of Lepidoptera) exist for the Northern Fennoscandia only. Fauna of the Finnish Lapland and the Kola Peninsula amounts around 700 species (Linnaluoto \& Koponen 1980, Kozlov \& Jalava 1994, Kozlov et al. 2000). Some 300 species have been recorded in Polar Ural ( $\mathrm{J}$. Kullberg, unpubl.). On the basis of this information we estimate that our list includes approximately $25-35 \%$ of the species that can be expected from the Taymyr national district. However, this estimate concerns mainly the southern parts of this region, south of the $70^{\circ} \mathrm{N}$; the diversity sharply declines to the north, and only seven specimens of four species of Lepidoptera have been collected during four summers in Severnaya Zemlya archipelago (O. Makarova, pers. comm.).

The present list includes only 56 species of the so-called "microlepidoptera" (36\% of the total list), while in the much better studied Kola Peninsula (Kozlov \& Jalava 1994, Kozlov et al. 2000, unpubl.) this group forms $62 \%$ the fauna. However, despite of the fragmentary character of the available material, several biogeographically important species were included. In particular, record of Greya variabilis in Taymyr is approx. 3,500 km to the East of the Provideniya Bay, the only locality from where this species had been reported in Palaearctic (Kozlov 1996). Argyroploce mengelana is new to East Palearctic region (so far known from North America and Novaya Zemlya), and Clepsis mehli was so far known only from Norway. These examples reveal our poor knowledge of the fauna of northern Siberia.

The fauna of Taymyr consists generally of boreal peat-bog and arctic or arctoalpine species, some of which (e.g. Colias hecla, C. tyche, Agriades glandon) have relict populations in Northern Fennoscandia which is separated from the continental arctic continuum by the White Sea. The largest part of Lepidoptera recorded in Taymyr (70 species; 45\%) is widely distributed, with 67 species having Holarctic range, and three being cosmopolitan (Plutella xylostella, Monopis weaverella, Vanessa cardui). Beringian and Palaearctic groups are represented by 30 and 31 species, respectively, and 24 species are restricted to the Eastern Palaearctic. Note that many of the so-called Beringian species are distributed far more to the West than the actual Beringia, which is located around both sides of the Beringian strait. The specialities of Taymyr are several high-arctic relict species restricted to the most 
arctic areas, such as Euxoa churchilliensis, Udea torvalis, Gynaephora rossii and Argyroploce mengelana. Thus, the fauna of moths and butterflies of Taymyr is clearly more similar to that of the East Palaearctic or even Polar Ural than to that of northwestern Europe. Many of the eastern species reach Urals mountains or the even more western Kanin peninsula. In an European perspective, this fauna looks quite exotic and may resemble the one that existed in Europe during the ice ages.

Acknowledgements. The collecting trip to Noril'sk was made possible by the financial support of the Academy of Finland (through the research exchange grant to $\mathrm{M}$. Kozlov), Natural History Museum in London, and Maj and Tor Nessling Foundation (Finland). We thank V. Zverev for assistance in collecting, V. Savchenko for practical arrangements, A. Lvovsky, S. Sinev, V. Kononenko, K. Eskov, V. Mironov and E. Belyaev for help in determination and providing us with information on published and unpublished records of different taxa from Taymyr, and $\mathrm{O}$. Karsholt for useful comments. Special thanks to the amateur lepidopterologists G. A. Grigorjev and B. A. Khramov (St. Petersburg), who allowed us to publish the data on the specimens from their private collections, and to O. L. Makarova for sending us the samples collected by researchers of A. N. Severtsov Institute of Ecology and Evolution (Moscow).

\section{References}

Aarvik, L. E. 2004: Tortricidae. — In: Karsholt, O. \& Nieukerken, E. J. van (eds.), Lepidoptera, Moths. Fauna Europaea version 1.1; available at http:// www.faunaeur.org

Antonova, E. M. 1976: (Butterflies of Taymyr.) — In: Biological problems of the North. VII Symposium. Invertebrate zoology, parasitology, physiology and biochemistry of animals: 6-7. Institute of Biology, Petrozavodsk [in Russian].

Chernov, Y. I. 1966: (Complex of flower visiting insects in tundra.) - Voprosy Geografii (Moscow) 69:76-97 [in Russian.]

Chernov, Y. I. 1973: (A review of trophic groups of invertebrates in typical tundra subzone of Western Taymyr.) - In: Tikhomirov, B. A. (ed.), Biogeocenoses of Taymyr tundra and their productivity, Part 2: 166-179. Nauka, Leningrad. [In Russian.]

Chernov, Y. I. 1978: (Structure of the animal population in the subarctic.) — Nauka, Moscow. 166 pp. [in Russian.]

Dubatolov, V. V., Chistyakov, Y. A. \& Ammosov, A. N. [1997] 1991: Tiger moths (Lepidoptera, Arctiidae: Arctiinae) of the North-East of the USSR. - In:
Matis, E. G. (ed.), Entomological studies in the NorthEast of the USSR, Part 2: 48-65. Vladivostok, Far East Branch of the Academy of Sciences of the USSR. [in Russian.]

Dubatolov, V. V. \& Zolotarenko, G. S. 1990: (On a fauna of tiger moths (Lepidoptera: Nolidae, Arctiidae) from the West Siberian plain.) — In: Zolotarenko, G. S. (ed.), Arthropods and helmints: 122-139. Novosibirsk, Nauka. [in Russian.]

Goater, B., Ronkay, L. \& Fibiger, M. 2003: Catocalinae \& Plusiinae. Noctuidae Europaeae, vol. 10. — Sorø, Entomological Press, $452 \mathrm{pp}$.

Gorbunov, P. Y. 2001: The butterflies of Russia: classification, genitalia, keys for identification (Lepidoptera: Hesperioidea and Papilionoidea). — Thesis, Ekaterinburg. 320 pp., 13 pls. [In Russian]

Karsholt, O. \& Razowski, J. (eds.) 1996: The Lepidoptera of Europe: A distributional checklist. - Apollo Books, Stenstrup, 380 p.

Klyuchko, Z. F. 1985: (Zonal and vertical distribution of Plusiinae (Lepidoptera).) — In: Zolotarenko, G. S. (ed.), Arthropods of Siberia and Far East: 183-191. Nauka, Novosibirsk. [in Russian.]

Klyuchko, Z. F. 1988: Specificity of distribution of Plusiinae (Lepidoptera, Noctuidae) of Siberia and Northern Europe. - In: Zlobin, V. V. (ed.), The connections between entomofauna of the Northern Europe and Siberia: 67-71. Zoological Institute, USSR Academy of Sciences, Leningrad. [in Russian.]

Kononenko, V. S. 1985: (Materials to the noctuid moths fauna (Lepidoptera, Noctuidae) of Upper Kolyma region.) - In: Zolotarenko, G. S. (ed.), Arthropods of Siberia and Far East: 168-182. Nauka, Novosibirsk. [in Russian.]

Kononenko, V. S. [1997] 1991: Materials on the noctuid fauna (Lepidoptera, Noctuidae) of Chukotka. - In: Matis, E. G. (ed.), Entomological studies in the NorthEast of the USSR, Part 2: 26-42. Vladivostok, Far East Branch of the Academy of Sciences of the USSR. [in Russian.]

Kononenko, V. S. 2005: An annotated check list of the Noctuidae (s. 1.) (Lepidoptera, Noctuoidea: Nolidae, Erebidae, Micronoctuidae, Noctuidae) of the Asian part of Russia and the Ural region. (Noctuidae Sibiricae. Vol. 1.) - Entomological Press, Sorø, 243 pp.

Kononenko, V. S., Lafontaine, J. D. \& Mikkola, K. 1996: Taxonomy and zoogeography of some arctic Noctuidae (Lepidoptera), with description of three new species and one new subspecies. - Acta Zoologica Fennica 200: 83-94.

Korshunov, Y. P. 1987: New taxones of lepidopterous [sic!] in Eurasia. - In: Zolotarenko, G. S. (ed.), Insects, mites and helmints. New and little known species of the fauna of Siberia 19: 9-13. Nauka, Novosibirsk. [in Russian.]

Korshunov, Y. P. 1996: Additions and corrections to the book: "Butterflies of Asian part of Russia". — ETA Grp. Publ., Novosibirsk, 66 pp. [in Russian.]

Korshunov, Y. P. 1998: New descriptions and adjustments 
for the book: "Butterflies of Asian part of Russia". Novosibirsk State Agrarian University, Novosibirsk, 71 pp. [in Russian.]

Korshunov, Y. P. 2000: Butterflies of Ural, Siberia and the Far East. Keys and annotations. - VITEL Publ., Novosibirsk, 192 pp. [in Russian.]

Korshunov, Y. P. 2002: Butterflies of North Asia. — KMK Press, Moscow, 424 pp. [in Russian.]

Korshunov, Y. \& Gorbunov, P. 1995: Butterflies of Asian part of Russia. A Guide. - Ural State University, Ekaterinburg, 202 pp. [in Russian.]

Korshunov, Y. P., Pupavkin, D. M. \& Chernenko, Y. I. 1982: Butterflies (Lepidoptera, Diurna) of transpolar Enissey and Taymyr. - In: Zolotarenko, G. S. (ed.), Beneficial and harmful insects of Siberia: 75-87. Nauka, Novosibirsk. [in Russian.]

Korshunov, Y. P., Elshin, S. V. \& Zolotarenko, G. S. 1985: Butterflies (Lepidoptera, Diurna) of Polar Ural, Yamal and Taymyr. - In: Zolotarenko, G. S. (ed.), Arthropods of Siberia and Far East: 93-105. Nauka, Novosibirsk. [in Russian.]

Kozlov, M. V. 1996: Incurvariidae and Prodoxidae (Lepidoptera) from Siberia and the Russian Far East, with descriptions of three new species. - Entomologica Fennica 7: 55-62.

Kozlov, M. V. \& Jalava, J. 1994: Lepidoptera of Kola Peninsula, Northwestern Russia. - Entomologica Fennica 5: $65-85$.

Kozlov, M. V., Jalava, J. \& Shutova, E. 2000: New records of Lepidoptera from the Kola Peninsula, Northwestern Russia. - Entomologica Fennica 11: 131-136.

Lafontaine, J. D, Mikkola, K. \& Kononenko, V. S. 1983: A revision of the genus Xestia subg. Schoyenia Auriv. (Lepidoptera, Noctuidae), with description of four new species and a new subspecies. - Entomologica Scandinavica 14: 337-369.

Lafontaine, J. D., Kononenko, V. S. \& McCabe, T. L. 1986: A review of the Lasionycta leucocycla complex (Lepidoptera, Noctuidae) with description of three new subspecies. - Canadian Entomologist 118: 255-279.

Lafontaine, J. D, Mikkola, K. \& Kononenko, V. S. 1987a: A revision of the genus Xestia subg. Pachnobia (Lepidoptera Noctuidae), with descriptions of two new subspecies. - Entomologica Scandinavica 18:305-331.

Lafontaine, J. D, Mikkola, K. \& Kononenko, V. S. 1987b: Anarta cordigera (Thunberg) (Lepidoptera: Noctuidae: Hadeninae), a species complex. - Canadian Entomologist 119: 931-940.

Lewaski, S. 1887: Ueber Argynnis polaris. - Societas Entomologica 2: 116.

Linnaluoto, E. T. \& Koponen, S. 1980: Lepidoptera of Utsjoki, northernmost Finland. - Kevo Notes 5: 168.

Lvovsky, A. L. 1984: Aporia crataegi (Linnaeus, 1758). - In: Gorodkov, K. B. (ed.), Provisional atlas of the insects of the European part of USSR. Maps 179-221: 46 (map 206). Nauka, Leningrad. [in Russian.]

Ménétriès, E. 1851: Insecten. — In: Middendorff, A. T. von (ed.), Reise in den äussersten Norden und Osten Sibiriens während der Jahre 1843 und 1844. Bd. 2.
Zoologie. Theil 1. Wirbellose Thiere: 45-76. Kaiserlichen Akademie der Wissenschaften, St. Petersburg.

Miller, W. E. \& Jalava, J. 2000: Boreal Olethreutini (Tortricidae): Wing and genitalia illustrations, a new synonymy, and new holarctic addition. - Journal of Lepidopterists' Society 54(2): 47-51.

Murzin, V. S. 1982a: Papilio machaon Linnaeus, 1758. In: Gorodkov, K. B. (ed.), Provisional atlas of the insects of the European part of USSR. Maps 126-178: 36 (map 158). Nauka, Leningrad. [in Russian.]

Murzin, V. S. 1982b: Colias palaeno Linnaeus, 1761. In: Gorodkov, K. B. (ed.), Provisional atlas of the insects of the European part of USSR. Maps 126-178: 37 (map 159). Nauka, Leningrad. [in Russian.]

Noril'sk 1999: [Noril'sk and surroundings: Atlas, scale 1:100,000]. - Military Cartography Enterprise, St. Petersburg, 104 pp. [in Russian.]

Pagenstecher, A. 1901: Die arktische Lepidopterenfauna. — In: Römer, F. \& Schaudinn, F. R. (eds.), Fauna Arctica, Bd. 2: 199-400. G. Fischer, Jena.

Sotavalta, O. 1962: Hyphoraia alpina Quens. (LEP., Arctiidae) rediscovered in Europe. - Annales Entomologici Fennici 28: 182-185.

Sotavalta, O. 1963: The generic position of Hyphoraia alpina Quens. (LEP., Arctiidae). — Annales Entomologici Fennici 29: 257-267.

Sotavalta, O. 1965: Studies on the variation of the wing venation of certain tiger moths (LEP., Arctiidae, subfam. Arctiinae). - Annales Academiae Scientiarum Fennicae. Series A. IV. Biologica. 42 p.

Syroechkovskij, E. E., Rogacheva, E. V., Syroechkovskij, E. E. jun., Kuvaev, V. B., Lappo, E. G., Romanenko, F. A., Khruleva, O. A., Chernov, Y. I., Chuprov, I. L. \& Chuprova, I. L. 2000: (Bolshoi Arkticheskij Nature Reserve.) - In: Shtilmark, F. R. (ed.), Nature Reserves of Siberia. Vol. 2: 56-81. Logata, Moscow. [in Russian.]

Times 2003: The Times comprehensive atlas of the world. 11th ed. - Times books, London, 544 pp.

Trybom, F. [1878] 1877: Dagfjarilar insamelade af svenske expeditionen till Jenisssei 1876. — Ofversigt of Kgl. Vetenskaps Akademien Forhandlingar 34(6): 35-51.

Tshistjakov, Y. A. 1990: (Pararctia tundrana sp. n. vicariant of P. subnebulosa (Dyar, 1899) (Lepidoptera, Arctiidae) in the Subarctic sector of Eurasia.) - In: Lelei, A. S. (ed.), Novosti sistematiki nasekomykh Dalnego Vostoka: 97-99. Institute of Biology and Soil Sciences, Vladivostok. [in Russian.]

Vasilenko, S. V. 1990: (Review of the species group Entephria polata Dup. (Lepidoptera, Geometridae).) - In: Zolotarenko, G. S. (ed.). Taxonomy of insects and helmints. New and little known species of the Siberian fauna 22: 79-89. Nauka, Novosibirsk. [in Russian.]

Vasilenko, S. V. 1995: Review of geometrid moths of the Xanthorhoe sajanaria group (Lepidoptera, Geometridae). - Entomologicheskoe Obozrenie 74: 662668. [in Russian.]

Viidalepp, J. 2001: Psychophora cinderella sp. n. - a new 
species of geometrid moth from Novaya Zemlya (Lepidoptera, Geometridae). - In: Sinev, S. Y. (ed.), Problems of systematics of the phytophagous Lepidoptera. Proceedings of Zoological Institute in St. Petersburg 291: 119-121. Zoological Institute, Russian Academy of Sciences, St. Petersburg. [in Russian.] Zagulajev, A. K. 1983: New and little known species of the moths (Lepidoptera: Tineidae, Micropterigidae, Pterophoridae) from the USSR and adjacent territo- ries. - Entomologicheskoe Obozrenie 62: 106-122. [in Russian.]

Zolotarenko, G. S. 1970: Cutworms of West Siberia. Nauka, Novosibirsk, 436 pp. [in Russian.]

Zolotarenko, G. S. 1990: (To the fauna noctuid moths (Lepidoptera, Noctuidae) of mountains of the Eastern Yakutia.) - In: Zolotarenko, G. S. (ed.), Chlenistonogie i gelminty: 185-203. Nauka, Novosibirsk. [in Russian.] 